\title{
Safety of subxiphoid uniportal video-assisted thoracoscopic surgery for anterior mediastinal tumour in obese patients
}

\author{
Weidong $\mathrm{Wu}^{1,2}$, Chun Chen ${ }^{1,2}$, Wei Zheng ${ }^{1,2}$, Lin Huang ${ }^{1}$, Pengqiang $\mathrm{Gao}^{2}$, Yong $\mathrm{Zhu}^{1,2}$ \\ ${ }^{1}$ Department of Thoracic Surgery, Fujian Medical University Union Hospital, Fuzhou, China \\ ${ }^{2}$ Fujian Key Laboratory of Cardio-Thoracic Surgery, Fujian Medical University, Fuzhou, China
}

Videosurgery Miniinv 2021; 16 (2): 377-381

DOI: https://doi.org/10.5114/wiitm.2020.100879

\begin{abstract}
Introduction: Subxiphoid uniportal video-assisted thoracoscopic surgery (VATS) has been gradually applied for the treatment of anterior mediastinal tumour (AMT). However, whether obesity is a risk factor for subxiphoid uniportal VATS for AMT is still unknown.

Aim: To explore the safety and short-term outcome of subxiphoid uniportal VATS for AMT in obese patients.

Material and methods: The clinical data of 142 patients who received VATS via subxiphoid approach for AMT were analysed. According to body mass index (BMI), the patients were divided into an obese group (BMI $\left.\geq 28 \mathrm{~kg} / \mathrm{m}^{2}\right)$ and a non-obese group $\left(B M I<28 \mathrm{~kg} / \mathrm{m}^{2}\right)$. Then, the clinical and surgical characteristics between the obese group and the non-obese group were analysed to explore the effect of obesity on VATS for AMT. The pain scores were evaluated by the Numeric Rating Scale.

Results: The operative time and tracheal intubation time using subxiphoid uniportal VATS for AMT in the obese group were longer than that in the non-obese group $(p<0.05)$. However, there was no obvious difference in intraoperative blood loss, chest tube drainage time, chest tube drainage volume, and length of hospital stay between the obese group and the non-obese group ( $p>0.05)$. Moreover, there was also no significant difference in postoperative complications, including pulmonary complications, wound infection, arrhythmia, and pulmonary leak, between the obese group and the non-obese group. In addition, the pain scores in the obese group were similar to those in the non-obese group. Conclusions: Although obesity might prolong operative time of subxiphoid uniportal VAST for AMT, it does not increase the rate of postoperative complications. An experienced centre can properly conduct VAST via subxiphoid approach when treating AMT in obese patients.
\end{abstract}

Key words: anterior mediastinal tumour, subxiphoid uniportal video-assisted thoracoscopic surgery, body mass index, obesity, postoperative complication

\section{Introduction}

Surgery is the principal treatment for patients with anterior mediastinal tumour (AMT) [1-3]. The good prognosis of patients with AMT depends on the completeness of resection. Thoracoscopic surgery has been widely used for the treatment of various thoracic diseases such as lung cancer [4], oesophageal cancer [5], and AMT [1] with the development of medical devices and techniques. Hsu first reported the case of thoracoscopic thymectomy via subxiphoid approach in 2002 and found that VATS via subxiphoid approach has several advantages including multi-angle surgical view, less trauma, less bleeding, shorter postoperative length of hospital stay, and faster postoperative recovery [6, 7]. Thus,

\section{Address for correspondence}

Yong Zhu MD, Department of Thoracic Surgery, Fujian Medical University Union Hospital, 29 Xinquan Road, Fuzhou 350001, China,

e-mail: dr.zhuyong@163.com 
subxiphoid uniportal video-assisted thoracoscopic surgery (VATS) has gradually been used more widely in the treatment of AMT.

As the prevalence of being overweight and obesity has grown over the past few decades $[8,9]$, more and more obese patients have received clinical surgery. Body mass index (BMI), which acts as an indicator of body fat, has been reported to be related with adverse outcomes, including metabolic, respiratory, cardiovascular, and musculoskeletal, which can adversely impact surgical outcomes to a certain extent [10]. Some researchers demonstrated that obesity is an important risk factor for postoperative complications, and the rate of postoperative complication in obese patients is higher than that in patients with normal weight [11-14]. However, some other researchers disagreed with that and pointed out that obesity did not affect postoperative complications in some surgery [15-18]. So far, whether a higher BMI can compromise surgical outcomes via subxiphoid uniportal VATS in patients with AMT is unclear.

In this retrospective study, 30 obese patients and 112 non-obese patients experiencing subxiphoid uniportal VATS for AMT were reviewed to determine the safety and usefulness of VATS via subxiphoid approach for AMT in obese patients.

\section{Aim}

The aim of present study was to explore the safety and short-term outcome of subxiphoid uniportal VATS for AMT in obese patients.

\section{Material and mthods}

\section{Patients}

A total of 142 patients with AMT were enrolled from July 2015 and July 2020. All of them were treated via subxiphoid uniportal VATS. These patients were retrospectively reviewed and analysed. The present study was approved by the local institutional review board. Patients were divided into two groups according to BMI: non-obese group (BMI $<28$ $\left.\mathrm{kg} / \mathrm{m}^{2}\right)$ and obese group $\left(\mathrm{BMI} \geq 28 \mathrm{~kg} / \mathrm{m}^{2}\right)$.

\section{Surgical technique}

Subxiphoid uniportal VATS was performed as described in previous literature [1, 19]. Briefly, the patient under general anaesthesia was placed in a lithotomy or supine position with their legs spread.
Then, a small transverse skin incision was typically made 1-2 cm below the lower edge of the xiphoid. GelPOINT ${ }^{\oplus}$ Mini (Applied Medical, Rancho Santa Margarita, CA, USA) was inserted and fixed at the incision site. Three subports were inserted into the GelPOINT ${ }^{\circledR}$ Mini platform. A camera scope and forceps were inserted into the three subports, and the operation was performed using only the subxiphoid incision.

\section{Pain management}

The pain scores were evaluated on the $3^{\text {rd }}, 7^{\text {th }}$, and $15^{\text {th }}$ day after operation by using the Numeric Rating Scale (NRS). All pain scores were recorded by clinical staff using NRS on an 11-point system ranging from 0 to 10 .

\section{Statistical analysis}

The data were analysed via GraphPad Prism 6 and SPSS 23.0 software. Mann-Whitney or Student's $t$-test were performed to analyse continuous variables. Fisher's exact or $\chi^{2}$ test were performed to analyse categorical variables. $P$-values $<0.05$ were considered significant.

\section{Results}

\section{Demographics}

Patient's demographic and clinical characteristics were described in Table I. A total of 142 patients with a mean age of 53.8 years (20-75 years), including 76 male patients and 65 female patients, were enrolled. The mean BMI was $25.1 \pm 3.5 \mathrm{~kg} / \mathrm{m}^{2}$. There were $30(21.1 \%)$ obese patients, and the remaining 112 (78.9\%) were non-obese. The mean BMI of obese group and non-obese were $29.2 \pm 2.4$ and 23.4 $\pm 1.3 \mathrm{~kg} / \mathrm{m}^{2}$. There was a higher rate of type II diabetes in the obese group compared with the non-obese group $(28.1 \%$ vs. $3.6 \%, p=0.02)$. There was no significant difference in gender, ages, the incidence of coronary heart disease, hypertension, and postoperative pathological types between the obese patients and non-obese patients.

\section{Analysis for surgical features}

The comparisons of surgical features between non-obese and obese groups were described in Table II. There was no obvious difference in intraoperative blood loss, chest tube drainage time, chest 
tube drainage volume, and length of hospital stay between the obese group and non-obese group $(p>0.05)$. Nonetheless, obese patients experienced significantly longer tracheal intubation time and operative time than non-obese ones $(p<0.05)$.

\section{Postoperative complications}

Postoperative complications are shown in Table III. There was no significant difference in the rates of postoperative complications between the obese group and non-obese group (12.7\% vs. $15.6 \%, p=$ $0.14)$. In addition, mean BMI was similar in patients with and without postoperative complications (24.78 \pm 2.9 vs. $\left.24.26 \pm 3.2 \mathrm{~kg} / \mathrm{m}^{2}, p=0.57\right)$. Seven (6.4\%) patients had postoperative pulmonary complications. Pulmonary complication rates were similar in the obese and non-obese groups (6.4\% vs. $6.3 \%, p=$ $0.73)$. We found no statistically significant increase in the odds ratio for complications in the obese group. In addition, the pain scores of 3 days, 7 days, and 15 days after operation also had no obvious difference between obese patients and non-obese patients $(p>0.05$, Table IV).

\section{Discussion}

Subxiphoid uniportal VATS has been widely used in different kinds of thoracic procedures, such as thymectomy, lobectomy, and resection of AMT [20]. Moreover, VAST is safe and reliable for large and invasive primary mediastinal tumours [21]. VATS via subxiphoid approach does not require sternotomy [22]. Thus, subxiphoid uniportal VATS is associated with less pain, less trauma, faster postoperative recovery, fewer complications, and better short-term and long-term outcomes [23, 24]. However, the use of subxiphoid uniportal VATS for AMT resection in obese patients has not been reported so far. Here-
Table I. Clinical features of patients in the nonobese group and obese group

\begin{tabular}{|c|c|c|c|}
\hline Variable & Non-obese & Obese & $P$-value \\
\hline Number & 110 & 32 & \\
\hline $\mathrm{BMI}\left[\mathrm{kg} / \mathrm{m}^{2}\right]$ & $23.4 \pm 1.3$ & $29.2 \pm 2.4$ & $<0.0001$ \\
\hline Gender: & & & 0.62 \\
\hline Male & 62 & 21 & \\
\hline Female & 48 & 11 & \\
\hline Age [years] & $55.82 \pm 9.72$ & $55.36 \pm 10.21$ & 0.74 \\
\hline Coronary heart disease: & 4 & 2 & 0.72 \\
\hline High blood pressure & 26 & 9 & 0.62 \\
\hline Type ii diabetes & 4 & 9 & 0.007 \\
\hline Myasthenia gravis & 12 & 5 & 0.76 \\
\hline $\begin{array}{l}\text { Postoperative } \\
\text { pathological types: }\end{array}$ & & & 0.71 \\
\hline Thymic cyst & 67 & 17 & \\
\hline Thymoma & 23 & 6 & \\
\hline Thymic hyperplasia & 8 & 2 & \\
\hline Others & 12 & 7 & \\
\hline
\end{tabular}

in, we described the safety of subxiphoid uniportal VATS for AMT in obese patients.

The potential effects of obesity on outcomes after surgery have received increasing attention [25, 26]. However, there is a paucity of data on the relationship between obesity and outcomes of subxiphoid uniportal VAST for AMT. With regard to the feasibility of VAST for obese patients, we conducted a retrospective study on 142 patients with AMT receiving subxiphoid uniportal VAST and found that VAST via subxiphoid approach for AMT in obese patients was safe and useful. In this study, we found that obese patients tended to have

Table II. Comparison of surgical features between obese group and non-obese group

\begin{tabular}{|c|c|c|c|}
\hline Variables & Non-obese $(n=110)$ & Obese $(n=32)$ & $P$-value \\
\hline Tracheal intubation time [s] & $32.12 \pm 8.76$ & $39.64 \pm 11.63$ & 0.002 \\
\hline Intraoperative blood loss [ml] & $27.63 \pm 18.93$ & $28.21 \pm 19.62$ & 0.63 \\
\hline Operative time [min] & $86.62 \pm 16.76$ & $95.32 \pm 17.53$ & 0.002 \\
\hline Chest tube drainage time [days] & $3.12 \pm 2.02$ & $2.63 \pm 0.67$ & 0.36 \\
\hline Chest tube drainage volume [ml] & $201.52 \pm 18.65$ & $203.63 \pm 19.52$ & 0.95 \\
\hline Length of hospital stay [days] & $3.56 \pm 1.68$ & $3.72 \pm 1.56$ & 0.86 \\
\hline
\end{tabular}


Table III. Postoperative complications in obese group and non-obese group

\begin{tabular}{|lccc|}
\hline Parameter & $\begin{array}{c}\text { Non- } \\
\text { obese } \\
(n=110)\end{array}$ & $\begin{array}{c}\text { Obese } \\
(n=32)\end{array}$ & $P$-value \\
\hline Complications (total) & 14 & 5 & 0.14 \\
\hline Pulmonary complications & 7 & 2 & 0.73 \\
\hline Wound infection & 2 & 1 & 0.62 \\
\hline Arrhythmia & 2 & 1 & 0.68 \\
\hline Pulmonary air leak & 3 & 1 & 0.56 \\
\hline
\end{tabular}

longer operative time. The operative time and anaesthesia time are important risk factors for postoperative complications. Patients with postoperative complications experienced significantly longer operative time than uncomplicated patients according to previous literature [27]. Thus, prolonged operative time may lead to a higher rate of postoperative complications, especially in elderly patients [28]. In the present study, although obese patients experienced longer operative time, there was no difference in postoperative complications between obese and non-obese patients. This may be due to the small increase in operative time in obese patients compared to non-obese patients. However, there was no significant difference between the two groups on pain score, which both still had a high level at 7 days and 15 days after surgery. We consider this for the following reasons. The incision was made in the thoracoabdominal junction which is usually related to great tension leading to the highest initial pain intensity. The post-operation analgesia method was set by flurbiprofen axetil via continuous infusion twice for 3 days followed by loxoprofen sodium via oral administration for 7 days. The analgesic effect of loxoprofen sodium was weaker than that of flurbiprofen axetil, so we noticed a slow decrease in pain intensity over time. Meanwhile, some discomfort during the hospital stay might have masked the pain, hence the pain score was still at a high level 7 days after surgery.
There are some limitations to this study. Firstly, the number of patients in the obese and non-obese groups was unequal. Secondly, the sample size was not large enough, and more patients should be enrolled in a following study. Thirdly, patients in this study were collected from a single centre, and multiple centres should be investigated for further studies. Lastly, the follow-up time was relatively short, and prolonged follow-up time should be performed to verify the present results.

\section{Conclusions}

Subxiphoid uniportal VATS for AMT does not increase the rate of postoperative complications in obese patients. VATS via subxiphoid approach is safe, efficient, and feasible and can provide good clinical outcomes for obese patients with AMT.

\section{Acknowledgments}

This work was supported by Joint Funds for the innovation of science and Technology-Fujian province (2018Y9017)

\section{Conflict of interest}

The authors declare no conflict of interest.

\section{References}

1. Numanami H, Yano M, Yamaji M, et al. Thoracoscopic thymectomy using a subxiphoid approach for anterior mediastinal tumors. Ann Thorac Cardiovasc Surg 2018; 24: 65-72.

2. Rakovich G, Deslauriers J. Video-assisted and minimally-invasive open chest surgery for the treatment of mediastinal tumors and masses. J Vis Surg 2017; 3: 25.

3. Shiomi K, Kitamura E, Ono M, et al. Feasible and promising modified trans-subxiphoid thoracoscopic extended thymectomy for patients with myasthenia gravis. J Thorac Dis 2018; 10: 1747-52.

4. Pardolesi A, Rolli L, Scanagatta P, et al. Comparison of clinical and oncologic effectiveness between flexible 3-dimensional and bidimensional video-thoracoscopic surgery for lung cancer. Tumori 2020 doi: 10.1177/0300891620925901.

5. He ZF, Zheng TL, Liu DL, et al. Comparison of short-term and long-term efficacy between robot-assisted and thoracosco-

Table IV. Postoperative pain score in obese and non-obese groups

\begin{tabular}{|lccc|}
\hline Postoperative pain score & Non-obese $(n=110)$ & Obese $(n=32)$ & $P$-value \\
\hline 3 days after surgery & $7.26 \pm 1.16$ & $7.32 \pm 1.24$ & 0.62 \\
\hline 7 days after surgery & $7.02 \pm 1.56$ & $6.86 \pm 1.48$ & 0.63 \\
\hline 15 days after surgery & $4.58 \pm 1.66$ & $4.64 \pm 1.84$ & 0.61 \\
\hline
\end{tabular}


py-laparoscopy-assisted radical esophageal cancer surgery. Zhonghua Wei Chang Wai Ke Za Zhi 2020; 23: 390-5.

6. Hsu CP. Subxiphoid approach for thoracoscopic thymectomy. Surg Endosc 2002; 16: 1105.

7. Suda T. Uniportal subxiphoid video-assisted thoracoscopic thymectomy. J Vis Surg 2016; 2: 123.

8. le Roux CW, Hartvig NV, Haase CL, et al. Obesity, cardiovascular risk and healthcare resource utilization in the UK. Eur J Prev Cardiol 2020 doi: 10.1177/2047487320925639.

9. Pajula S, Jyranki J, Tukiainen E, et al. Complications after lower body contouring surgery due to massive weight loss unaffected by weight loss method. J Plast Reconstr Aesthet Surg 2019; 72: 649-55.

10. Borracci RA, Ingino CA, Miranda JM. Association of body mass index with short-term outcomes after cardiac surgery: retrospective study and meta-analysis. Medicina (B Aires) 2018; 78 171-9.

11. Zhang ZJ, Zheng ML, Nie Y, et al. Comparison of Arndt-endobronchial blocker plus laryngeal mask airway with left-sided double-lumen endobronchial tube in one-lung ventilation in thoracic surgery in the morbidly obese. Braz I Med Biol Res 2017; 51: e6825.

12. Zogg CK, Mungo B, Lidor AO, et al. Influence of body mass index on outcomes after major resection for cancer. Surgery 2015; 158: 472-85.

13. Wang BS, Ma RZ, Liu YQ, et al. Body mass index as an independent risk factor for inferior vena cava resection during thrombectomy for venous tumor thrombus of renal cell carcinoma. World J Surg Oncol 2019; 17: 17.

14. Qiu Y, Liu Q, Chen G, et al. Outcome of rectal cancer surgery in obese and nonobese patients: a meta-analysis. World I Surg Oncol 2016; 14: 23.

15. Liu M, Xing J, Arslan A, et al. Safety and efficacy of laparoscopic gastrectomy in obese patients with gastric cancer. Medicine (Baltimore) 2019; 98: e17991.

16. Yang $\mathrm{H}$, Xing J, Cui M, et al. Efficacy evaluation of laparoscopy-assisted radical gastrectomy in obese patients with gastric cancer. Zhonghua Wei Chang Wai Ke Za Zhi 2014; 17: 776-80.

17. Tsujiura M, Hiki N, Ohashi M, et al. Should pylorus-preserving gastrectomy be performed for overweight/obese patients with gastric cancer? Gastric Cancer 2019; 22: 1247-55.

18. Chen H, Sui W. Influence of obesity on short- and long-term outcomes after laparoscopic distal gastrectomy for gastric cancer. J BUON 2017; 22: 417-23.

19. Suda T. Subxiphoid uniportal video-assisted thoracoscopic surgery procedure. Thorac Surg Clin 2017; 27: 381-6.

20. Luo M, Kong D. Subxiphoid uniportal video-assisted thoracoscopic surgery: a cosmetically superior approach to submammary rib tumor resection. Thorac Cancer 2019; 10: 2316-8.

21. Fang Y, Qin Z. Video-assisted thoracoscopic surgery is safe and reliable for large and invasive primary mediastinal tumors. Videosurgery Miniinv 2020. DOI: https://doi.org/10.5114/ wiitm.2020.94528.

22. Li J, Qi G, Liu Y, et al. Meta-analysis of subxiphoid approach versus lateral approach for thoracoscopic thymectomy. J Cardiothorac Surg 2020; 15: 89 .
23. Yano M, Moriyama S, Haneda $\mathrm{H}$, et al. The subxiphoid approach leads to less invasive thoracoscopic thymectomy than the lateral approach. World J Surg 2017; 41: 763-70.

24. Abu-Akar F, Gonzalez-Rivas D, Yang C, et al. Subxiphoid uniportal VATS for thymic and combined mediastinal and pulmonary resections - a two-year experience. Semin Thorac Cardiovasc Surg 2019; 31: 614-9.

25. Wang Z, Zhang X, Liang J, et al. Short-term outcomes for laparoscopy-assisted distal gastrectomy for body mass index >/=30 patients with gastric cancer. J Surg Res 2015; 195: 83-8.

26. Horii N, Sawda Y, Kumamoto T, et al. Impact of intramuscular adipose tissue content on short- and long-term outcomes of hepatectomy for colorectal liver metastasis: a retrospective analysis. World J Surg Oncol 2020; 18: 68.

27. Sugimoto M, Kinoshita T, Shibasaki H, et al. Short-term outcome of total laparoscopic distal gastrectomy for overweight and obese patients with gastric cancer. Surg Endosc 2013; 27: 4291-6.

28. Sun L, Zhao B, Huang Y, et al. Feasibility of laparoscopy gastrectomy for gastric cancer in the patients with high body mass index: a systematic review and meta-analysis. Asian J Surg 2020; 43: 69-77.

Received: 27.08.2020, accepted: 23.09.2020. 